\title{
Challenges in translation: models to promote translation
}

Jürgen Popp

Dennis Matthews

Alfredo Martinez-Coll

Thomas Mayerhöfer

Brian C. Wilson 


\title{
Challenges in translation: models to promote translation
}

\author{
Jürgen Popp, ${ }^{\mathrm{a}, \star}$ Dennis Matthews, ${ }^{\mathrm{b}}$ Alfredo Martinez-Coll, ${ }^{\mathrm{c}}$ Thomas Mayerhöfer, ${ }^{\mathrm{d}}$ and Brian C. Wilson ${ }^{\mathrm{e}}$ \\ aLeibniz Institute of Photonic Technology, Jena, Germany \\ bUniversity of California, Davis, California, United States \\ 'MTPConnect, Sydney, New South Wales, Australia \\ dLeibniz Institute of Photonic Technology, Jena, Germany \\ eUniversity Health Network/University of Toronto, Toronto, Ontario, Canada
}

\begin{abstract}
We first discuss the main obstacles for clinical translation of biophotonics technologies, in particular, the different valleys of death, after which we present different examples of ways to bridge these gaps, remove the obstacles, and promote clinical translation. @ 2017 Society of Photo-Optical Instrumentation Engineers (SPIE) [DOI: 10.1117/1.JBO.23.2 .021101]
\end{abstract}

Keywords: clinical translation; biophotonics technologies; models to promote translation.

Paper 170464SSR received Jul. 15, 2017; accepted for publication Sep. 21, 2017; published online Oct. $12,2017$.

\section{Obstacles to Clinical Translation}

Biophotonics is a multidisciplinary field aiming to prevent, diagnose, and cure diseases with optical technologies and, at the same time, it is a business sector with double digit growth rates. Biophotonics can be provisionally subdivided in the areas of imaging technologies, point-of-care techniques, and therapy approaches, each of which comprises a multitude of different technologies and approaches. Out of these many approaches, only a small percentage have been advanced into medical products and have made it into routine clinical practice, for example, optical coherence tomography (OCT) in ophthalmology, ${ }^{1}$ fluorescence imaging in endoscopy, ${ }^{2}$ and fluorescence-based immunoassays for point-of-care applications. ${ }^{3}$ What are the obstacles that obviously prevent biophotonics technologies, in particular in-vivo-techniques, from their translation to the clinics and how can these obstacles be removed?

First of all, there is no lack of basic and applied research. ${ }^{4}$ Indeed, for the medical sector in general, including biophotonics, only a small fraction of the research efforts leads to final products: "in 2008, more than 800,000 medical research papers were published but by 2011 , only 21 new drugs had been approved by the US Food and Drug Administration." the demand side, there is also no lack of need for medical innovations. On the contrary, in particular, our aging societies long for ways to increase the quality of life in old age and to fight age-related diseases. Medical insurance companies actively look for possibilities to reduce costs while, at the same time, trying to satisfy their customers' and the patients' demands. However, while large research efforts exist and there is no lack of demand, the challenges in the chain from basic and applied research to a product or service that is reimbursable by the medical insurance companies are numerous. ${ }^{6-8}$

*Address all correspondence to: Jürgen Popp, E-mail: juergen.popp@ leibniz-ipht.de
A first challenge lies in the fact that many of the researchers have neither time, knowledge, the resources or the budget to further develop an idea to a final product. Quite often, they have to move on once the principal feasibility (i.e., the proofof-concept) of their idea is shown. Taking the US as a typical example, funding by the National Science Foundation to those from other sources like the Engineering Research Centers, the Partnerships for Innovation: Building Innovation Capacity and finally Innovation Corps and Small Business Innovation Research and Small Business Technology Transfer programs only cover research to the proof-of-concept stage and to earlystage prototype. Product development and commercialization has to be funded from private funds. In addition, for clinical researchers, research activities concerning the development of new technologies are generally not equally valued compared to clinical work on patients, which rewards the latter more than research.

Overall, in a very simplified model, the way to the final product can be compartmentalized into three different phases. Phase one comprises the idea, its implementation, and proof-of-concept [technology readiness level (TRL) 1 to 3, cf. 4)]. As already pointed out, this phase is where academic researchers are mostly involved. Since, ideally, a medical need is the starting point, in this phase, mainly clinicians or medical doctors and researchers are involved. The next phase is more engineering than research (product design and development). Accordingly, the main part of the work is carried out by engineers, but the medical enduser plays an important role in defining the demands, as in this phase the instrumentation has to be prepared to leave the lab and enter the clinics. Accordingly, everything needs to be shrunk down to fit into a housing which needs to be compact and robust enough to be brought to the clinics (TRL 4 to 6). Besides safety considerations, ergonomics, user friendliness, and efficacy also begin to play more important roles toward clinical trials, the third and final phase in which these properties of the product have to be demonstrated. In this phase, naturally, the main part of the work is carried out by the clinicians. As potential examples, where development might have followed these stages, technologies developed by multidisciplinary centers like the 
Beckman Laser Institute might be mentioned, e.g., Doppler OCT for imaging blood flow. ${ }^{7}$

Before the clinical trials can begin, however, institutional and regulatory approval has to be obtained. In terms of logistics and financial resources, this stage usually exceeds by far what academic groups can muster. Since new technology is usually considered very risky, it will not easily be taken up by industry or commercial investors. Typically, investors tend to derisk investments by outsourcing development-related work to research groups or start-ups. New technology is often acquired by big companies by buying start-ups, but seldom before successful clinical trials. On the other hand, forming a start-up just to get the industry interested in a new technology creates an even larger overhead of work for an academic team. Furthermore, quite often, approvals for the use of experimental devices in humans comprise manufacture of such devices under design control protocols and compliance with quality assurance practices. In the usual case, universities have neither established good manufacturing practice protocols compliant with quality systems regulations for medical devices nor dedicated prototyping areas. A final point in this regard is the establishing of intellectual property, which is costly and an additional burden on the way to a product, where, quite often, the budget will be extremely strained by concept development.

With regard to the regulatory hurdles, it must also be stated that it is hard to demonstrate that a new device or technique is safe, when, in reality, safety standards still require clarification, such as in, e.g., safety standards for optical radiation. In this particular case, the current safety standards have been transferred blindly from those obtained by studies of eye and skin exposure to optical radiation delivered to internal tissues, which are usually not exposed to light and might, therefore, be more sensitive to it. Again, the conduct of such safety studies is way beyond what a typical research group can accomplish.

Clinical trials also represent a critical stage that is usually not financed by any photonics-related governmental funds. In addition, very close collaboration with clinicians is needed. In the ideal case, this collaboration should already exist at the stage of the idea, which should be based on a significant unmet medical need. Clinicians should also give advice on important considerations at the engineering step, since, e.g., hygienic considerations might be very important to select proper instrument housing, while ergonomics for the clinician and patient might be different from what a researcher understands by that term. Once the regulatory approval has been granted and clinical trials begin, the new device or technology has not only to demonstrate its benefit for the patient but also needs to show that it can be seamlessly integrated into clinical practice and also has benefits for the clinical team. These benefits have to be convincing, as people working in the healthcare sector, in particular clinicians, are necessarily conservative ${ }^{9}$ and will not be easily convinced of the new technique or procedure, when they do not fully understand the risks or the potential benefits.

But even after this is achieved, success is not guaranteed. A further hurdle remains, namely, reimbursement: gaining of acceptance by the health insurance companies and/or the public healthcare providers to pay for the device. ${ }^{10}$ Without it, no standard procedure employing the new device or technique will be adopted by doctors or clinics.

Indeed, translation of medical research into a medical product is much more complicated than that of any other kind of research. Nevertheless, there are possible ways to bridge this extraordinarily broad valley of death, which will be discussed in Sec. 2.

\section{Ways to Bridge the Valley(s) of Death}

One of the new photonics-based techniques that has made its way into the clinics is OCT. Could OCT serve as an example from which general rules could be derived? Probably yes, even if in hindsight, it seems to be serendipitous that OCT succeeded, including the fact that many components used in OCT originally stem from the telecommunications industry. Since these components were already in use in nonhealth-related products, substantial effort and resources had already been invested to make them compact, robust, and relatively low cost. Another factor that played a role in OCT's success is that it has much in common with ultrasound, which is a very well-established technology in medical practice. Hence, clinicians find OCT relatively easy to use. Furthermore, the information provided by OCT is straightforward to understand, while its higher resolution over ultrasound is usually greatly appreciated. OCT also benefitted from fulfilling a variety of different clinical needs in other specialties after it had already been established in ophthalmology.

Nevertheless, it must also be stated that, despite all of these favorable circumstances, the further development of OCT into a product was quite often touch-and-go. At several points, Carl Zeiss Meditec AG (the developing company) was close to giving up on OCT. ${ }^{11}$

Because of the 2008 financial crisis, but also due to new problematic developments like the Theranos debacle, ${ }^{12}$ the lack of appetite for risk taking has increased the difficulties in obtaining the venture capital that is urgently needed for clinical translation of new technologies. Therefore, there is an evident need for a much more targeted approach. Such an approach should start with a dedicated team of scientists and clinicians matching specific unmet clinical needs with existing and emerging technologies. These teams should seek out companies interested in further developing the technology at as early a stage as possible. Certainly, there needs to be matched financial resources from appropriate funders to succeed and make it through the development phases and clinical trials. It is further of upmost importance that healthcare providers and insurance companies are engaged at the earliest possible stages to guarantee that the clinical use of the evolving instruments, methods, and procedures will be reimbursable.

This is an idea that is pursued, for example, at InfectoGnostics, which is a research campus funded for 15 years and set up 5 years ago in Jena, Germany, and which is a kind of onestop shop along the whole value creation chain. It comprises different laboratories with a plethora of photonic instrumentation and with access to clinical samples and clinical experts. In addition, it offers specialized services for technology transfer and scientific steering and networking from an idea to a product and potential market entry. Furthermore, it offers mentoring by experienced entrepreneurs as well as support for start-up companies. These measures are flanked by specialized education programs, e.g., a master of medical photonics, which can be pursued by students with a natural science or medical background.

To further strengthen these activities, Jena is trying to establish a Leibniz Center for Photonics specializing in infection research (LPI). The LPI pursues the aim of combating infectious diseases by means of photonics-based diagnostic tools and therapeutic procedures. From the technological side, the photonics 
technology platform will comprise state-of-the-art time and space-resolved spectroscopic imaging as well as innovative multimodal imaging technologies spanning the whole spectral range from extreme ultraviolet to the far infrared, and photonic molecular-biological point-of-care technologies. These technology platforms will enable diagnosis and treatment, including the treatment control of difficult-to-treat infections, as well as infections in the context of immunosuppression and defined comorbidities. The photonic technologies will be combined with biomedical technologies from high dimensional biology (i.e., genomics, transcriptomics, proteomics, and metabolomics), next generation sequencing and enabling technologies, such as microfluidics and chemometrics, in order to use these directly in a multidisciplinary manner in clinical settings.

Innovative diagnostic technologies and therapeutic approaches are to be created using a comprehensive innovation pipeline -starting with the concept and extending through to the validation procedure-in the form of a completely innovative research infrastructure open to national and international users, for whom LPI will provide, as a onestop agency under S2 and S3 safety conditions, a complete diagnostic and therapeutic service pipeline in order to develop their proof-of-concepts further. In the diagnostics pipeline, LPI will provide the necessary infrastructure in the form of modules, such as "photonics technologies," "target identification," "assay development," "sample center/biobank," etc. At a certain stage of the R\&D, the LPI researchers will develop, together with guest researchers and industrial partners, a diagnostic prototype, for which there will be a designated module called "design freeze." The prototype will then be tested and validated. By means of flying study nurses, the diagnostic and therapeutic approaches researched and implemented in the form of prototypes will be put into practice at various hospital sites and, thereby, directly subjected to multicenter clinical validation. All these facilities will be perfectly intertwined to further develop an idea toward instruments that serve the aim of clinicians and the patient as well as possible.

Of course, the LPI is just one idea that can be taken as an example ready to be transferred to other locations and other techniques. Nevertheless, overall, it is important that such and other initiatives join forces on several levels. Such an idea, not only for biophotonics but also for all technologies, is being pursued by the European Commission under the Pan-European initiative "digital innovation hubs."13 Under a digital innovation hub (DIH), the European Commission means "a one-stop shop that help companies to become more competitive with regard to their business/production processes, products, or services using digital technologies." These innovation hubs will be funded according to the European commission: "it is the ambition of the Commission to focus 500M€ over the next 5 years from Horizon 2020 budget to these actions" and aim to help derisk innovation for companies: "with the rapid pace of change in digital technologies, most decision makers in industry have difficulties to decide when to invest, up to what level and in which technologies."

The idea of the innovation hubs is to connect different competence centers that, very much like the planned LPI, are able to provide a technological infrastructure or a technology platform together with knowledge and expertise in different fields "to support their customers with piloting, testing, and experimenting with digital innovations." The latter also means that the competence center supports experimentation in a business environment and is able to demonstrate best practice. Such competence centers already exist in different countries all over Europe and for different fields. The term "competence center" is actually quite broadly defined. According to the European Commission, the DIHs are regional multipartner corporations that can include organizations like "research and technology organizations, universities, industry associations, chambers of commerce, incubator/accelerators, regional development agencies, and even governments, and can also have strong linkages with service providers outside of their region supporting companies with access to their services."

The idea behind the DIH is to make better use of this already existing expertise and equipment. One of its tasks is, therefore, to develop and support an ecosystem which "can help ensure that every company, small or large, high-tech or not, can grasp the digital opportunities," to which photonics technologies, including biophotonics, belong. Therefore, a DIH also supports brokerage, helps to access funds, and market intelligence. Beyond that, they also support training and education to help to establish the workforce necessary for innovation. Furthermore, they also provide incubator services, which mean that proximity is considered a very crucial factor. DIHs are meant as first regional contact points.

A somewhat different approach is being pursued in Australia, where, for example, MTPConnect ${ }^{14}$ serves as the Industry Growth Centre for MedTech and Pharmaceuticals to connect researchers with investors and companies to support translation. In addition, MTPConnect has close contacts with Industry Associations, regulators and government as well as governmentbased funding initiatives. As one of six industry growth centers, MTPConnect supports regulatory reforms, helps to gain improved access to global supply chains and international markets, and improves the engagement between research and business as well as industry-specific management and workforce skills. MTPConnect's activities are based on a "10-year sector competitiveness plan"15 with "a vision and strategy to maximize the sector's competiveness and productivity, and to help it achieve more rapid and sustained growth." Its goal is to establish Australia as a hub for medical technology, biotechnology, and pharmaceutical companies. Of its seven sector growth priorities, there are two of special interest for translation in biophotonics, namely "create a highly productive commercialization environment from research to early clinical trials and proof-of-concept" as well as "support the development of digitally enabled MTP solutions: devices and data analytics." Overall, MTPConnect's role is threefold. On the one hand, MTPConnect acts as an independent voice that, based on intimate knowledge of the sector, will help to change it toward obtaining sustainable growth. In addition, it will proactively "foster collaboration and competition, educate sector participants, and aggregate existing knowledge." A third activity of MTPConnect is that it acts as a funding entity, which targets industry-led and dollar-for-dollar matched projects that aim to fill gaps identified in the sector (around policy, regulation, funding and focus, commercialization and collaboration, skills, and global supply chain). Overall, MTPConnect's vision is to act as the nexus for all sector stakeholders, to create a collaborative culture, and to assist in building a robust ecosystem for the efficient translation of medical and pharmaceutical innovations in Australia.

While the innovation hubs that will be initiated in Europe and MTPConnect focus more on connecting already existing approaches or initiating new ones, InfectoGnostics together with the 
planned Leibniz Center for Photonics specialized in infection research are more similar to existing multidisciplinary approaches like the well-known Wellman Center for Photomedicine (Boston, United States), the Beckman Laser Institute and Medical Clinic (Irvine, United States) as well as the Medical Laser Center Lübeck. ${ }^{7}$ While the LPI's focus is with infectious diseases on a different specialty, it shares with the aforementioned institutions important features like "intellectual diversity" concentrated in one center, the possibility to carry out clinical studies and to "supporting high-risk, high-reward research.", What is different though, is that it is designed as a research infrastructure, so that external research groups can also profit from its capacities. Overall, we think what is generally important is that we need much more of such multidisciplinary initiatives not only to cover the broadness of biophotonics as a field and to take all chances that biophotonics technologies offer, but also to see which approach works best.

In conclusion, while there exist major challenges to enable a better translation of biophotonics research into the clinic, several new initiatives are on the way to help to remove the existing barriers together with future ones. Nevertheless, these initiatives can only support individual enterprises, so that it is of utmost importance that all involved stakeholders continuously work on an improvement of the current situation in order to serve the enduser of the biophotonics technologies and, ultimately, the patient.

\section{Disclosures}

The authors have no relevant financial interests in this article and no potential conflicts of interest to disclose.

\section{References}

1. https://www.zeiss.com/meditec/us/products/oct-optical-coherencetomography.html.

2. https://www.karlstorz.com/de/en/fi.htm.

3. http://www.alere.com/en/home/product-details/afinion-as100-analyzerus.html.

4. Note that we use terms like basic and applied research, proof-of-concept etc. in analogy to the technology readiness levels (TRLs) as e.g., defined by the European commission. Basic and applied research would be TRL 1-3, proof-of-concept TRL 3, early-stage prototype TRL 5-6, European Commission, Horizon 2020-Work Programme 2014-2015, General Annexes, http://ec.europa.eu/research/participants/data/ref/h2020/wp/ 2014_2015/annexes/h2020-wp1415-annex-g-trl_en.pdf.

5. "Crossing the valley of death: effective administrative support needed," Tomorrow's Medicine Issue, https://www.singhealth.com. sg/TomorrowsMed/Article/Pages/CrossingthevalleyofdeathEffective administrativesupportneeded.aspx (9 February 2015).

6. J. Popp, "Label-free photonic imaging modalities: overcoming barriers to clinical translation," BioPhotonics, January 2017, https://www. photonics.com/Article.aspx?AID=61449.

7. B. J. Tromberg et al., "Biomedical optics centers: forty years of multidisciplinary clinical translation for improving human health," J. Biomed. Opt. 21(12), 124001 (2016).
8. G. Apiou-Sbirlea et al., "Anatomy and physiology of translation: the academic research imperative," Clin. Invest. 5, 797-804 (2015).

9. D. Jemielniak and J. Kociatkiewicz, Management Practices in HighTech Environments, IGI Publishing, Hershey, Pennsylvania (2008).

10. S. D. Reed, A. M. Shea, and K. A. Schulman, "Economic implications of potential changes to regulatory and reimbursement policies for medical devices," J. Gen. Intern. Med. 23(Suppl. 1), 50-56 (2008).

11. P. French et.al., "Label-free optical techniques for biomedical diagnostics \& imaging: challenges and opportunities for clinical translation," http://www.osa.org/osaorg/media/osa.media/Meetings/PDFSupporting Doc/OSA_Label-free_diagnostics_white_paper.pdf (2016).

12. J. Carreyrou, "Hot Startup theranos has struggled with its blood-test technology," https://www.wsj.com/articles/theranos-has-struggled-withblood-tests-1444881901 (16 October 2015).

13. http://s3platform.jrc.ec.europa.eu/digital-innovation-hubs.

14. www.mtpconnect.org.au.

15. https://www.mtpconnect.org.au/Category?Action=View\&Category_ id $=125$.

Jürgen Popp studied chemistry at the Universities of Erlangen and Würzburg. Since 2002, he has held a chair for physical chemistry at Friedrich-Schiller University Jena. Since 2006, he has also been a scientific director of the Leibniz Institute of Photonic Technology, Jena. His research interests are mainly concerned with biophotonics in particular developing and employing innovative Raman techniques. He has published more than 630 journal papers and is founding editor and editor-in-chief of the Journal of Biophotonics.

Dennis Matthews is the emeritus director of the NSF Center for biophotonics at the University of California at Davis and an emeritus professor in the Department of Neurological Surgery. His current interests are in developing optical, RF, and x-ray technologies for disease diagnosis and treatment. He is a fellow of the American Physical Society, the Society of Photo and Industrial Engineers and the Optical Society of America and has more than 300 publications and 35 patents.

Alfredo Martinez-Coll obtained his BS in biomedical engineering, a $\mathrm{PhD}$ in near-infrared spectroscopy, and postdoctoral training in biomedical sciences. He brings more than 16 years research experience in cardio/cerebrovascular physiology, membrane transport, and oxygen transport to tissue. For the last 13 years, he has been working in technology transfer and commercialization at public hospitals and academia focusing on entrepreneurship and innovation, project management, and business development; joining MTPConnect to assist translation of research discoveries into social impact.

Thomas Mayerhöfer obtained his diploma in chemistry in 1996 at the University of Regensburg and his PhD at Friedrich-Schiller University in Jena in 1999. In 2006, he finished his habilitation titled "Optics and IR-spectroscopy of polydomain materials" in physical chemistry. Afterward, he was network support officer von Photonics4Life, the Network of Excellence in Biophotonics. Scientifically, he is working in the fields of optics in spectroscopy and, recently, of plasmonicenhanced vibrational spectroscopy.

Brian C. Wilson is a professor of medical biophysics, University of Toronto and Princess Margaret Cancer Centre, where he directs a multidisciplinary program in the development and clinical translation of optical and nanotechnologies, primarily for cancer imaging and treatment. 